\title{
Impact of prophylactic unilateral central neck dissection needed for patients with papillary thyroid microcarcinoma
}

\author{
Young Jae Ryu, Jung Han Yoon \\ Department of Surgery, Chonnam National University Medical School, Hwasun-gun, Jeonnam, Korea \\ Contributions: (I) Conception and design: JH Yoon; (II) Administrative support: YJ Ryu; (III) Provision of study materials or patients: All authors; (IV) \\ Collection and assembly of data: All authors; (V) Data analysis and interpretation: YJ Ryu; (VI) Manuscript writing: All authors; (VII) Final approval \\ of manuscript: All authors. \\ Correspondence to: Young Jae Ryu. Department of Surgery, Chonnam National University Medical School, 322 Seoyang-ro Hwasun-eup, Hwasun- \\ gun, Jeonnam 58128, Korea. Email: brandon-surgery@hotmail.com.
}

\begin{abstract}
Background Papillary thyroid microcarcinoma (PTMC), the increase in the diagnosis of which has led to an overall rise in the diagnostic rate of thyroid malignancies, is generally managed through a reduction in the surgical extent. Nevertheless, a considerable number of patients with PTMC experience subclinical central lymph node (LN) metastases following prophylactic central neck dissection (CND). This study aimed to investigate the impact of prophylactic CND on locoregional recurrence in PTMC patients who underwent hemithyroidectomy.

Methods: We reviewed the medical records of 1,071 patients with clinically LN-negative PTMC who underwent hemithyroidectomy between 2004 and 2012. Cox proportional hazards regression analysis was performed to investigate the predictive factors for recurrence. The median follow-up duration was 79 months (range, 12-176 months).
\end{abstract}

Results: Totally, 613 patients underwent hemithyroidectomy only, whereas 458 underwent hemithyroidectomy plus prophylactic unilateral CND. Recurrence was observed in 27 patients (eight and 19 patients in the prophylactic and non-prophylactic CND groups, respectively). Patients with a tumor size $\leq 6 \mathrm{~mm}$ (hazard ratio, 2.927; 95\% confidence interval, 1.372-6.245; P=0.005) had favorable recurrence-free survival (RFS); however, there was no relationship between prophylactic unilateral CND and RFS.

Conclusions: The incidence of locoregional recurrence was low in patients with PTMC who underwent hemithyroidectomy. In addition, prophylactic unilateral CND performance was not associated with RFS in PTMC. Accordingly, the use of prophylactic unilateral CND for clinically LN-negative PTMC should be avoided.

Keywords: Hemithyroidectomy; papillary thyroid carcinoma (PTC); prophylactic central neck dissection (CND); recurrence

Submitted Nov 29, 2019. Accepted for publication Feb 14, 2020.

doi: 10.21037 /gs.2020.03.03

View this article at: http://dx.doi.org/10.21037/gs.2020.03.03

\section{Introduction}

Papillary thyroid carcinoma (PTC) is the most commonly occurring thyroid malignancy. Its incidence has been increasing with the high detection rate of papillary thyroid microcarcinoma (PTMC), characterized by a maximum tumor size $\leq 1 \mathrm{~cm}$, through high-resolution ultrasonography
(US)-guided fine-needle aspiration cytology (FNAC). The reduction in the surgical extent for PTMC has shifted because lobectomy is suitable for PTC patients with a tumor size $<4 \mathrm{~cm}$ (1). Active surveillance has emerged as an alternative option for low-risk PTMC (2). Accordingly, mutual communication between physicians and patients is important in the selection of active surveillance 
versus surgery. Minimization of the surgical extent and performance of appropriate follow-up may be reasonable for patients who have anxiety regarding active surveillance.

Although LN involvement is commonly observed in patients with PTC at the time of the first diagnosis (3), the detection rate of $\mathrm{LN}$ involvement using preoperative imaging modalities is lower in the central compartment than in the lateral neck compartment, owing to the anatomical location (air-filled trachea and the thyroid gland itself). In addition, the use of prophylactic central neck dissection (CND) for patients with clinically LNnegative PTC is debatable. Although bilateral prophylactic CND is helpful in decreasing the rate of locoregional recurrence (4), it could be associated with increases in the rate of postoperative complications $(5,6)$. Complications occurring after the resection of thyroid gland and LNs are not commonly observed in patients who have undergone thyroid lobectomy or hemithyroidectomy, possibly because the contralateral thyroid gland may not have been evaluated. It is necessary to determine the efficacy of prophylactic unilateral CND in terms of locoregional recurrence. However, few studies have evaluated the impact of prophylactic unilateral CND on PTMC in cases with hemithyroidectomy. Therefore, the current study aimed to assess the relationship between prophylactic unilateral $\mathrm{CND}$ and recurrence-free survival (RFS) and to evaluate the prognostic factors associated with recurrence in patients with PTMC who had undergone hemithyroidectomy.

\section{Methods}

\section{Study population}

We reviewed the electronic medical records of 5,448 patients with PTMC who had undergone thyroid surgery at Chonnam National University Hwasun Hospital between 2004 and 2012. Patients were excluded if they met the following criteria: had undergone total or neartotal thyroidectomy, were followed-up for $<1$ year, had experienced recurrence within 6 months because of the possibility of residual or persistent disease, had pathologic results confirming disease other than classical PTC, had secondary malignancies preoperatively or postoperatively, had contralateral thyroid nodules, had clinically positive $\mathrm{LN}$ in the central or lateral neck compartment, had abnormal thyroid function test results, and had missing clinicopathological values. A total of 1,071 patients with PTMC who had undergone hemithyroidectomy with or without prophylactic unilateral CND were enrolled. This retrospective study was approved by the institutional review board of Chonnam National University Hwasun Hospital, and the need for informed consent from patients was waived. Data on the patients' clinicopathological characteristics, postoperative complications, and recurrence were collected.

\section{Surgery and pathology}

All suspicious thyroid nodules were evaluated via FNAC. In order to evaluate the $\mathrm{LN}$ involvement in the central or lateral neck compartment, all patients underwent preoperative US and computed tomography (CT). Hemithyroidectomy included resection of one lobe of the thyroid, isthmus, and pyramidal lobe. Prophylactic unilateral CND was performed at the surgeon's discretion. CND involved removal of the pretracheal, paratracheal, and prelaryngeal LNs. Endoscopic surgery was performed via the bilateral axillo-breast approach. Experienced pathologists measured the tumor size using surgical specimens. Chronic lymphocytic thyroiditis (CLT) was defined as the presence of diffuse lymphocytic infiltration in the surgical specimen. Minimal extrathyroidal extension (ETE) was defined as cancer extending into or just beyond the thyroid capsule, but not into any structures in the neck. The $\mathrm{N}$ stage of patients who underwent only hemithyroidectomy was $\mathrm{Nx}$, whereas the $\mathrm{N}$ stage of patients who underwent prophylactic CND was classified as $\mathrm{N} 0$ and $\mathrm{N} 1$ a according to $\mathrm{LN}$ involvement.

\section{Postoperative follow-up}

All patients were followed-up at the hospital every 6 months for the first 2 years and then annually thereafter. Patients who were followed-up for longer than 10 years underwent regular check-ups every 2 years. Physical examinations, thyroid function tests [triiodothyronine, free thyroxine, and thyroid stimulating hormone (TSH)], thyroglobulin $(\mathrm{Tg})$ and anti-Tg antibody levels tests, and US were performed at every visit. CT was selectively performed for patients with increasing levels of $\mathrm{Tg}$ or anti-Tg antibody irrespective of whether structural disease was observed on US during the follow-up. Locoregional recurrence was defined as the presence of visible disease in the remnant thyroid, central compartment, and lateral compartment. All structural recurrences were confirmed using US-guided FNAC. 
Table 1 Patients' demographic characteristics $(n=1,071)$

\begin{tabular}{|c|c|}
\hline Variable & Value \\
\hline \multicolumn{2}{|l|}{ Age (years), n (\%) } \\
\hline$\leq 55$ & $965(90.1)$ \\
\hline$>55$ & $106(9.9)$ \\
\hline \multicolumn{2}{|l|}{ Sex, n (\%) } \\
\hline Male & $217(20.3)$ \\
\hline Female & $854(79.7)$ \\
\hline \multicolumn{2}{|l|}{ Underlying disease, $\mathrm{n}(\%)$} \\
\hline Diabetes/hypertension & $25(2.3) / 104(9.7)$ \\
\hline \multicolumn{2}{|l|}{ Preoperative TSH level (mU/L), n (\%) } \\
\hline$\leq 2.5$ & $815(76.1)$ \\
\hline$>2.5$ & $256(23.9)$ \\
\hline \multicolumn{2}{|l|}{ Tumor size (mm), n (\%) } \\
\hline$\leq 6$ & $799(74.6)$ \\
\hline$>6$ & $272(25.4)$ \\
\hline \multicolumn{2}{|l|}{ N stage, n (\%) } \\
\hline $\mathrm{Nx}$ & $613(57.2)$ \\
\hline No & $363(33.9)$ \\
\hline $\mathrm{N} 1 \mathrm{a}$ & $95(8.9)$ \\
\hline Multifocality, n (\%) & $72(6.7)$ \\
\hline CLT, n (\%) & $212(19.8)$ \\
\hline Minimal ETE, n (\%) & $55(5.1)$ \\
\hline Lymphovascular invasion, n (\%) & $5(0.5)$ \\
\hline \multicolumn{2}{|l|}{ Method of surgery, n (\%) } \\
\hline Open & $893(83.4)$ \\
\hline Endoscopic & $178(16.6)$ \\
\hline Recurrence, n (\%) & $27(2.5)$ \\
\hline Median follow-up period, months & 79 (range, 12-176) \\
\hline
\end{tabular}

$\mathrm{TSH}$, thyroid stimulating hormone; ETE, extrathyroidal extension; CLT, Chronic lymphocytic thyroiditis.

\section{Postoperative complications}

Hypocalcemia was defined as the presence of a below-normal total calcium level (reference range, $8.0-10.2 \mathrm{mg} / \mathrm{dL}$ ) regardless of the parathyroid hormone level. Hypoparathyroidism was defined as the presence of a below-normal postoperative serum parathyroid hormone level (reference range, $8-51 \mathrm{pg} / \mathrm{mL}$ ) with a concomitant low calcium level. The total and ionized calcium levels were examined along with the parathyroid hormone level at 6 hours, 1 day, and 2 days after surgery. Patients who had developed hypoparathyroidism were prescribed calcium carbonate and calcitriol depending on their individual needs. Flexible laryngoscopy was not indicated for all patients for the evaluation of vocal cord palsy; however, patients with suspicions of recurrent laryngeal nerve (RLN) injury during surgery or who had developed voice changes postoperatively underwent flexible laryngoscopy. Hypoparathyroidism and RLN injury were classified as transient if the associated symptoms resolved within 6 months after surgery; they were classified as permanent if the associated symptoms resolved after more than 6 months. Postoperative bleeding was defined as bleeding requiring surgical intervention.

\section{Statistical analysis}

RFS was defined as the time between surgery completion and the occurrence of recurrent disease. Continuous variables were presented as median (range) or mean (standard deviation), whereas categorical variables were presented as number (percentage). When comparing patients according to $\mathrm{CND}$, an independent $t$-test was used for continuous variables and a chi-square test for categorical variables. The optimal cutoff tumor size was calculated using receiver operating characteristic curves. A univariate Cox proportional hazards model was used to analyze the relationship between clinicopathological variables and RFS. Multivariate Cox proportional hazards regression was performed using the backward elimination method. All statistical analyses were performed using SPSS version 23.0 (IBM Inc., Armonk, NY, USA). Statistical significance was defined as $\mathrm{P} \leq 0.05$.

\section{Results}

\section{Patient demographics}

Table 1 shows the clinicopathological characteristics of all patients. Of the 1,071 patients who underwent thyroid hemithyroidectomy, 217 (20.3\%) were men. A total of 613 patients underwent hemithyroidectomy only $(\mathrm{Nx}$ stage, i.e., the no CND group), whereas 458 underwent hemithyroidectomy plus prophylactic unilateral CND (i.e., the CND group). Of the patients who underwent CND, 363 (33.9\%) had N0 stage disease and 95 (8.9\%) had confirmed N1a stage disease. Of the 1,071 patients, 19 and eight patients in the no CND group and CND group, 


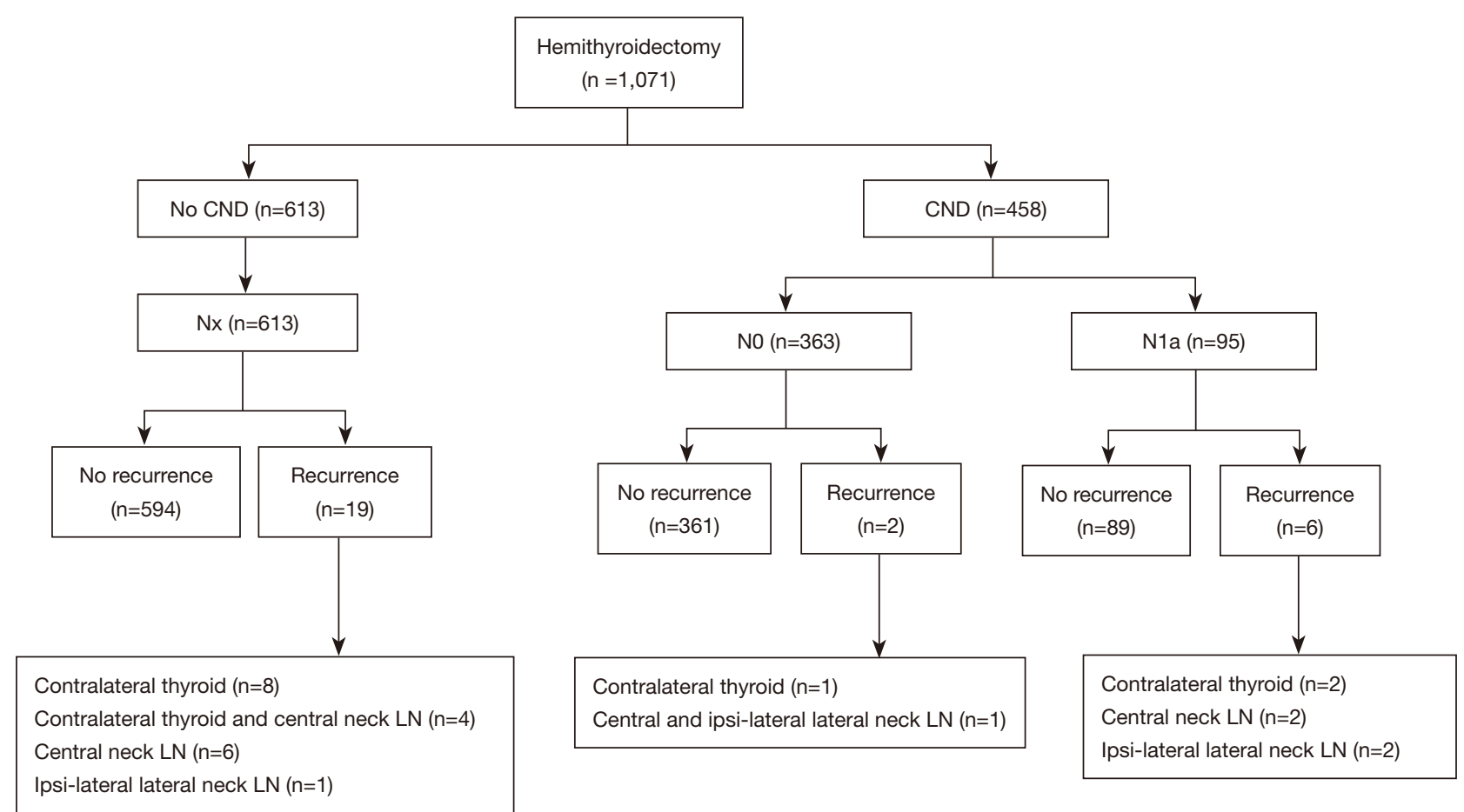

Figure 1 Distribution of recurrence during follow-up. CND, central neck dissection; LN, lymph node.

respectively, developed recurrence. Of the 27 patients with recurrence during the follow-up period, 21 showed recurrent disease within 5 years after the initial surgery. Recurrent lesions were most commonly observed in the contralateral thyroid. Lateral neck $\mathrm{LN}$ recurrences were ipsilateral primary tumors. None of the patients developed distant metastases (Figure 1).

\section{Comparison according to prophylactic unilateral CND}

Patients in the CND group tended to have larger tumors ( $\leq 6$ vs. $>6 \mathrm{~mm}, \mathrm{P}=0.004)$ and CLT as the result of pathologic analysis $(\mathrm{P}<0.001)$. The mean numbers of harvested and metastatic LNs in the CND group were $3.23 \pm 2.05$ and $0.32 \pm 0.79$, respectively. N0 stage disease was observed in 363 patients and N1a stage in 95 patients. Patients in the CND group tended to undergo open surgery (Table 2).

\section{Univariate and multivariate analyses according to recurrence}

Univariate analysis revealed that a tumor size $\leq 6 \mathrm{~mm}$ ( $v s$. $>6 \mathrm{~mm}, \mathrm{P}=0.008$ ) was associated with better RFS. However, the performance of unilateral CND did not decrease the rate of recurrence. In addition, patient age, sex, preoperative TSH level, multifocality, CLT, minimal ETE, and the method of surgery were not correlated with a better RFS. In the multivariate analysis, patients with a tumor size $\leq 6 \mathrm{~mm}$ [hazard ratio (HR), 2.927; 95\% confidence interval (CI), 1.372-6.245; $\mathrm{P}=0.005$ ] had favorable RFS (Table 3).

\section{Postoperative complications}

Transient hypoparathyroidism was observed in 10 patients (6 in the no CND group and 4 in the CND group), whereas permanent hypoparathyroidism was observed in one patient in the no CND group. Transient RLN palsy was observed in 28 patients (18 in the no CND group and 10 in the CND group) and permanent RLN palsy, in two patients (one each in the CND and no CND groups). Postoperative bleeding was observed in two patients in the no CND group who were treated with surgery (Table 4).

\section{Discussion}

In the current study, 20.7\% (95/458) of patients with PTMC who had undergone hemithyroidectomy plus prophylactic unilateral CND showed subclinical LN 
Table 2 Comparison of variables according to CND and LN status

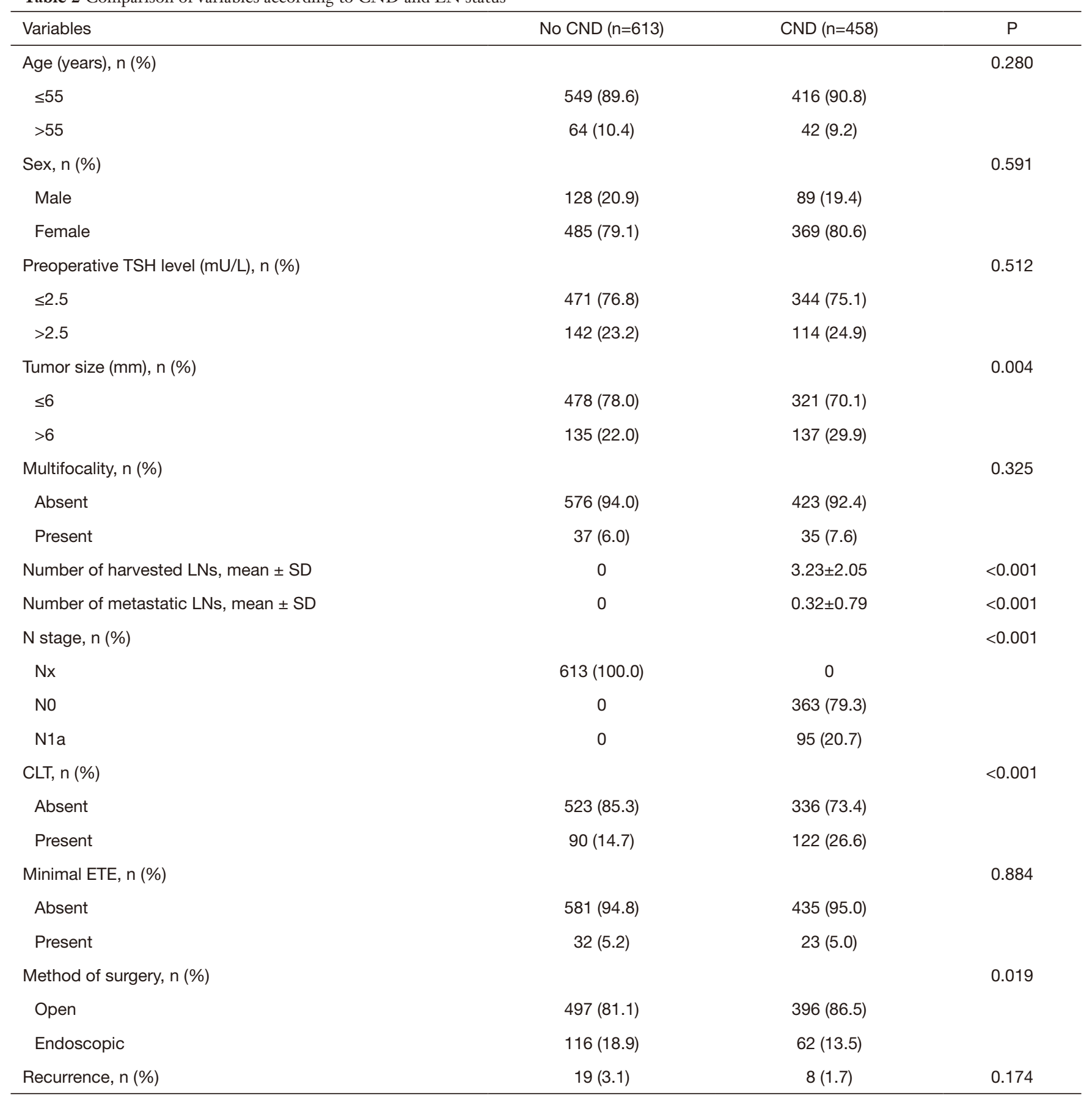

CND, central neck dissection; LN, lymph node; TSH, thyroid stimulating hormone; CLT, chronic lymphocytic thyroiditis; ETE, extrathyroidal extension.

metastases. Although different recurrence rates were observed according to whether prophylactic unilateral CND had been performed, prophylactic unilateral CND was insufficient for patients with PTMC and clinically LN- negative disease. Surgery is essential for PTC management; however, according to American Thyroid Association (ATA) guidelines, active surveillance could be an option for patients with clinically $\mathrm{LN}$-negative disease without local 
Table 3 Univariate analysis according to recurrence

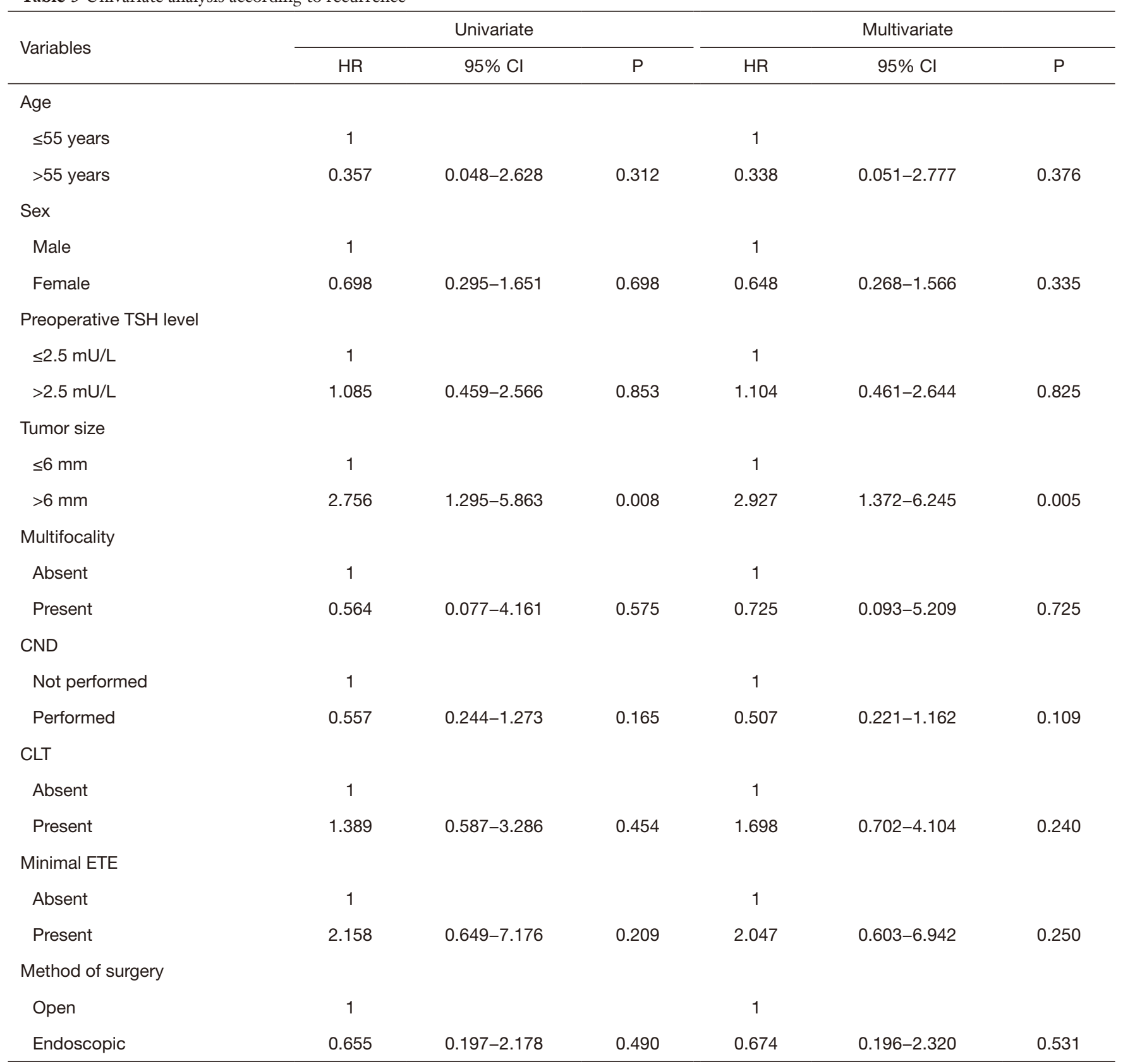

$\mathrm{HR}$, hazard ratio; $\mathrm{Cl}$, confidence interval; TSH, thyroid stimulating hormone; CND, central neck dissection; CLT, Chronic lymphocytic thyroiditis; ETE, extrathyroidal extension.

invasion and less aggressive PTMC (1). However, longterm follow-up after surgery is more cost-effective than long-term active surveillance (7). In addition, minimization of the surgical extent and performance of appropriate follow-up could be helpful for patients who may be stressed about undergoing active surveillance.
Small thyroid nodules are frequently diagnosed as PTMC through high-resolution US-guided FNAC, and the increase in the PTMC diagnosis rate has contributed to an overall increase in the rate of thyroid malignancies. The early diagnosis of tiny thyroid nodules as PTMC can lead to a reduction in the surgical extent. A recent 
Table 4 Postoperative complications

\begin{tabular}{lcc}
\hline Complications & No CND $(\mathrm{n}=613)$ & $\mathrm{CND}(\mathrm{n}=458)$ \\
\hline Hypoparathyroidism, $\mathrm{n}(\%)$ & & $4(0.9)$ \\
Transient & $1(0.0)$ & 0 \\
Permanent & & $10(2.2)$ \\
RLN injury, $\mathrm{n}(\%)$ & $18(2.9)$ & $1(0.2)$ \\
Transient & $1(0.2)$ & 0 \\
Permanent & $2(0.3)$ & $1 \%$ \\
Postoperative bleeding, $\mathrm{n}(\%)$ & & \\
\hline
\end{tabular}

CND, central neck dissection; RLN, recurrent laryngeal nerve.

meta-analysis revealed that patients with PTMC who had undergone lobectomy had mortality rates that were similar to those among patients who had undergone total thyroidectomy, although the results suggested that lobectomy is more suitable for patients with PTMC (8). More attention should be paid to recurrence in patients with PTMC. Patients with PTMC who had undergone hemithyroidectomy and unilateral CND had a lower rate of locoregional recurrence than did those who had undergone hemithyroidectomy only (9). In contrast, concomitant unilateral CND with hemithyroidectomy was not associated with superior disease-free survival rate than that on hemithyroidectomy only (10). The present study found recurrence rates of $2.5 \%$ and $1.7 \%$ in the no CND and CND groups, respectively. Although prophylactic unilateral CND improved the recurrence rate by $0.8 \%$, it was not associated with a better RFS rate. In the present study, the LN status of patients who had not undergone prophylactic CND was heterogeneous. Therefore, it is difficult to conclude that among the clinically LN-negative PTMC cases all patients who had undergone hemithyroidectomy had a true LN-negative status. Accordingly, accurate LN staging via prophylactic unilateral CND could be helpful in decreasing the rate of recurrence; however, unilateral CND performance is not associated with lower RFS rates.

Patients who had undergone total thyroidectomy because of unilateral PTMC could show possible occult PTMC in the contralateral thyroid lobe; therefore, total thyroidectomy is appropriate for patients with multifocal tumors in one lobe or benign nodules in the contralateral thyroid gland even in the case of PTMC $(11,12)$. However, another study demonstrated that lobectomy was the safe initial treatment for low-risk small PTC with non-suspicious contralateral thyroid nodules (13). No randomized trial has compared the survival outcomes in patients with PTMC considering the surgical extent of total thyroidectomy and hemithyroidectomy. In a study of PTMC that evaluated patients' long-term postoperative outcomes, no difference in the recurrence rate between patients who had undergone total thyroidectomy and those who had undergone lobectomy was found (14). Patients with PTMC who had undergone lobectomy with prophylactic unilateral CND had long-term rates of death and locoregional recurrence that were similar to those among patients who had undergone total thyroidectomy and prophylactic bilateral CND for PTMC; hence, complete thyroidectomy should be limited to patients with recurrence (15). Furthermore, patients with PTMC who had undergone total thyroidectomy did not experience the positive effects of radioactive iodine therapy (16). Hemithyroidectomy reduces the occurrence rate of morbidities such as hypoparathyroidism and RLN injury through omission of exploration of the contralateral thyroid gland, avoidance of overtreatment with radioactive iodine therapy, and minimization of or abstinence from levothyroxine use. Furthermore, locoregional recurrence was detected early using postoperative biochemical information and US (17). Similar to the results obtained in previous studies $(12,18)$, the contralateral thyroid gland was found to be the most frequently observed site of recurrence in the present study. Hence, contralateral thyroid nodules should be carefully observed during the follow-up of patients with PTMC who undergo hemithyroidectomy. In the present study, no complications were observed after reoperation in patients with structural disease.

In a previous study, a preoperative TSH level $>2.5 \mathrm{mU} / \mathrm{L}$ was found to be an independent predictor of central LN metastases in PTMC (19). In another study, patients with TSH levels $>1.85 \mathrm{mU} / \mathrm{L}$ at 1 year after surgery had higher 
rates of recurrence (20). According to ATA guidelines, the TSH level should be maintained at 0.5 to $2.0 \mathrm{mU} / \mathrm{L}$ for patients who underwent lobectomy (1). In the current study, the TSH suppression strategy was not used in all patients. Levothyroxine was administered for maintenance of TSH levels within the normal range and for alleviation of hypothyroidism-related symptoms. Although the preoperative TSH level was not associated with RFS in the current study, further studies should evaluate the impact of TSH levels on recurrence in patients with PTMC who have undergone hemithyroidectomy.

Larger primary PTC tumor sizes are associated with more frequent detection of cervical LN metastasis at first diagnosis (21). In particular, patients with PTMC may show lateral neck LN metastasis if the tumor is located in the upper thyroid pole, invades the adjacent capsule, or is accompanied by central neck LN metastasis $(22,23)$. Thus, the tumor size at diagnosis influences the surgical extent as well as survival outcomes despite PTMC being considered a small thyroid cancer. Among patients with PTMC, primary tumor sizes $>7 \mathrm{~mm}$ were associated with aggressive features such as central LN metastases, capsular invasion, and ETE (24). Different tumor sizes are associated with recurrence among PTMC patients who underwent hemithyroidectomy (10). In the current study, we calculated the optimal cutoff tumor size for the prediction of recurrence using receiver operating characteristic curves. Although prophylactic unilateral CND was often performed for patients with a primary tumor size $>6 \mathrm{~mm}$, tumor sizes $>6 \mathrm{~mm}$ were significantly associated with lower RFS.

This study has some limitations. The study was retrospective in nature, was performed at a single institution, and included relatively short-term follow-up periods in some patients. Although all patients had clinically LN-negative PTMC, prophylactic unilateral CND was performed at the surgeons' discretion. Moreover, although US is usually used for the detection of structural recurrence, some patients had undergone CT. Furthermore, patients with unilateral multifocal PTMC generally underwent total thyroidectomy according to our institution's policy. Therefore, selection bias may have occurred.

\section{Conclusions}

Although the management of PTMC is shifting toward minimization of the surgical extent, many patients who had undergone prophylactic unilateral CND were confirmed as having subclinical LN metastases in our study.
Determining the LN status via prophylactic unilateral CND should have resulted in better RFS rates; however, the use of prophylactic unilateral CND was not found to be associated with a better RFS rates. In fact, most cases of recurrent disease that were observed in the remnant thyroid were treated with reoperation, without complications, in PTMC patients who had undergone hemithyroidectomy. Therefore, our results suggested that prophylactic unilateral CND should be avoided for PTMC patients in whom hemithyroidectomy is planned; moreover, careful examination after surgery should be performed in patients with a tumor size $>6 \mathrm{~mm}$.

\section{Acknowledgments}

Funding: This study was funded by Chonnam National University Hospital Biomedical Research Institute (grant number HCRI 19021).

\section{Footnote}

Conflicts of Interest: Both authors have completed the ICMJE uniform disclosure form (available at http://dx.doi. org/10.21037/gs.2020.03.03). The authors have no conflicts of interest to declare.

Ethical Statement: The authors are accountable for all aspects of the work in ensuring that questions related to the accuracy or integrity of any part of the work are appropriately investigated and resolved. The study was approved by the institutional review board of Chonnam National University Hwasun Hospital (CNUHH-2019-130), and the need for informed consent from patients was waived.

Open Access Statement: This is an Open Access article distributed in accordance with the Creative Commons Attribution-NonCommercial-NoDerivs 4.0 International License (CC BY-NC-ND 4.0), which permits the noncommercial replication and distribution of the article with the strict proviso that no changes or edits are made and the original work is properly cited (including links to both the formal publication through the relevant DOI and the license). See: https://creativecommons.org/licenses/by-nc-nd/4.0/.

\section{References}

1. Haugen BR, Alexander EK, Bible KC, et al. 2015 
American Thyroid Association Management Guidelines for Adult Patients with Thyroid Nodules and Differentiated Thyroid Cancer: The American Thyroid Association Guidelines Task Force on Thyroid Nodules and Differentiated Thyroid Cancer. Thyroid 2016;26:1-133.

2. Miyauchi A. Clinical Trials of Active Surveillance of Papillary Microcarcinoma of the Thyroid. World J Surg 2016;40:516-22.

3. So YK, Son YI, Hong SD, et al. Subclinical lymph node metastasis in papillary thyroid microcarcinoma: a study of 551 resections. Surgery 2010;148:526-31.

4. American Thyroid Association Guidelines Taskforce on Thyroid N, Differentiated Thyroid C, Cooper DS, et al. Revised American Thyroid Association management guidelines for patients with thyroid nodules and differentiated thyroid cancer. Thyroid 2009;19:1167-214.

5. Ywata de Carvalho A, Chulam TC, Kowalski LP. Longterm Results of Observation vs Prophylactic Selective Level VI Neck Dissection for Papillary Thyroid Carcinoma at a Cancer Center. JAMA Otolaryngol Head Neck Surg 2015;141:599-606.

6. Zhao W, You L, Hou X, et al. The Effect of Prophylactic Central Neck Dissection on Locoregional Recurrence in Papillary Thyroid Cancer After Total Thyroidectomy: A Systematic Review and Meta-Analysis: pCND for the Locoregional Recurrence of Papillary Thyroid Cancer. Ann Surg Oncol 2017;24:2189-98.

7. Lin JF, Jonker PKC, Cunich M, et al. Surgery alone for papillary thyroid microcarcinoma is less costly and more effective than long term active surveillance. Surgery 2020;167:110-6.

8. Zheng W, Li J, Lv P, et al. Treatment efficacy between total thyroidectomy and lobectomy for patients with papillary thyroid microcarcinoma: A systemic review and meta-analysis. Eur J Surg Oncol 2018;44:1679-84.

9. Hyun SM, Song HY, Kim SY, et al. Impact of combined prophylactic unilateral central neck dissection and hemithyroidectomy in patients with papillary thyroid microcarcinoma. Ann Surg Oncol 2012;19:591-6.

10. Son HJ, Kim JK, Jung YD, et al. Comparison of outcomes between hemithyroidectomy alone and hemithyroidectomy with elective unilateral central neck dissection in patients with papillary thyroid microcarcinoma. Head Neck 2018;40:2449-54.

11. Koo BS, Lim HS, Lim YC, et al. Occult contralateral carcinoma in patients with unilateral papillary thyroid microcarcinoma. Ann Surg Oncol 2010;17:1101-5.

12. Kim SK, Park I, Woo JW, et al. Total thyroidectomy versus lobectomy in conventional papillary thyroid microcarcinoma: Analysis of 8,676 patients at a single institution. Surgery 2017;161:485-92.

13. Ritter A, Bachar G, Hirsch D, et al. Natural History of Contralateral Nodules After Lobectomy in Patients With Papillary Thyroid Carcinoma. J Clin Endocrinol Metab 2018;103:407-14.

14. Brito JP, Hay ID. Management of Papillary Thyroid Microcarcinoma. Endocrinol Metab Clin North Am 2019;48:199-213.

15. Lee J, Park JH, Lee CR, et al. Long-term outcomes of total thyroidectomy versus thyroid lobectomy for papillary thyroid microcarcinoma: comparative analysis after propensity score matching. Thyroid 2013;23:1408-15.

16. Hu G, Zhu W, Yang W, et al. The Effectiveness of Radioactive Iodine Remnant Ablation for Papillary Thyroid Microcarcinoma: A Systematic Review and Metaanalysis. World J Surg 2016;40:100-9.

17. Gibelli B, Dionisio R, Ansarin M. Role of hemithyroidectomy in differentiated thyroid cancer. Curr Opin Otolaryngol Head Neck Surg 2015;23:99-106.

18. Kwon H, Jeon MJ, Kim WG, et al. A comparison of lobectomy and total thyroidectomy in patients with papillary thyroid microcarcinoma: a retrospective individual risk factor-matched cohort study. Eur J Endocrinol 2017;176:371-8.

19. Gao Y, Qu N, Zhang L, et al. Preoperative ultrasonography and serum thyroid-stimulating hormone on predicting central lymph node metastasis in thyroid nodules as or suspicious for papillary thyroid microcarcinoma. Tumour Biol 2016;37:7453-9.

20. Park JH, Lee YM, Lee YH, et al. The prognostic value of serum thyroid-stimulating hormone level post-lobectomy in low- and intermediate-risk papillary thyroid carcinoma. J Surg Oncol 2018;118:390-6.

21. Gomez NR, Kouniavsky G, Tsai HL, et al. Tumor size and presence of calcifications on ultrasonography are pre-operative predictors of lymph node metastases in patients with papillary thyroid cancer. J Surg Oncol 2011;104:613-6.

22. Kwak JY, Kim EK, Kim MJ, et al. Papillary microcarcinoma of the thyroid: predicting factors of lateral neck node metastasis. Ann Surg Oncol 2009;16:1348-55. 
23. Jeon MJ, Chung MS, Kwon H, et al. Features of papillary thyroid microcarcinoma associated with lateral cervical lymph node metastasis. Clin Endocrinol (Oxf) 2017;86:845-51.

Cite this article as: Ryu YJ, Yoon JH. Impact of prophylactic unilateral central neck dissection needed for patients with papillary thyroid microcarcinoma. Gland Surg 2020;9(2):352-361. doi: $10.21037 /$ gs.2020.03.03
24. Lee KJ, Cho YJ, Kim SJ, et al. Analysis of the clinicopathologic features of papillary thyroid microcarcinoma based on 7-mm tumor size. World J Surg 2011;35:318-23. 